\title{
Artificial Intelligence Alongside Physicians in Canada: Reality and Risks
}

\section{Sumedha Sachar ${ }^{1,2 *}$, Maïa Dakessian ${ }^{1,3^{*}}$, Saina Beitari ${ }^{1,4^{*}}$, Saishree Badrinarayanan ${ }^{1,5^{*}}$}

*All authors contributed equally

${ }^{1}$ Science \& Policy Exchange Canada

2Department of Pharmacology, Université de Montréal, Monteal, QC

${ }^{3}$ Département de médecine sociale et préventive, Université de Montréal, Montreal, QC

${ }^{4}$ Department of Microbiology and Immunology, McGill University, Montreal, QC

5Integrated Program in Neuroscience, McGill University, Montreal, QC

http://doi.org/10.38126/ISPG170119

Corresponding author: saishree.badrinarayanan@mail.mcgill.ca

Keywords: healthcare; artificial intelligence; machine learning; regulations; data privacy; interoperability; digital health; PIPEDA; GDPR

Executive Summary: Artificial intelligence (AI) and machine learning (ML) have the potential to revolutionize the healthcare system with their immense potential to diagnose, personalize treatments, and reduce physician burnout. These technologies are highly dependent on large datasets to learn from and require data sharing across organizations for reliable and efficient predictive analysis. However, adoption of AI/ML technologies will require policy imperatives to address the challenges of data privacy, accountability, and bias. To form a regulatory framework, we propose that algorithms should be interpretable and that companies that utilize a black box model for their algorithms be held accountable for the output of their ML systems. To aid in increasing accountability and reducing bias, physicians can be educated about the inherent bias that can be generated from the ML system. We further discuss the potential benefits and disadvantages of existing privacy standards ((Personal Information Protection and Electronic Documents Act) PIPEDA and (Personal Information Protection and Electronic Documents Act) GDPR) at the federal, provincial and territorial levels. We emphasize responsible implementation of AI by ethics, skill-building, and minimizing data privacy breaches while boosting innovation and increased accessibility and interoperability across provinces.

\section{Statement of the issue}

Machine learning is an application of AI that provides systems the ability to learn from data, classify, predict, and improve itself automatically without being explicitly programmed (Luxton 2016). These technologies are poised to revolutionize the healthcare world by assisting physicians in diagnosis, treatment (Martin 2019; New 2018), prognosis, and early intervention (O'Kane 2018) of certain diseases and health conditions. A timely example is the Canadian start-up BlueDot, first to predict the outbreak of the COVID-19 pandemic, which is now helping track and contain the virus as a part of the
Canadian national task force (Steig 2020). Harnessing automation, with the help of chatbots and telemedicine (Siwicki 2020) to support the overwhelmed healthcare system in this crisis can improve treatment by enhancing accessibility to patients and may also reduce healthcare costs in the long term (Health Analytics 2020).

For instance, it has been suggested that optimizing innovation and building new tools for physicians and consumers could generate $\$ 100$ billion per year in the US (Catell et al 2013). Nevertheless, further research is needed to fully assess the economic benefits of the 
use of these systems (Wolff et al 2020), and there are still other challenges that require attention. Currently in Canada the absence of a national framework that allows for health records to be portable across provinces hinders the quality of care patients receive (Goldman 2019). For digital interoperability (the ability to exchange data across health systems)(Lehne et al 2019) to succeed, a comprehensive framework on privacy policies is required (Brady 2020).

A central Canadian policy framework that was established in 2000, the Personal Information Protection and Electronic Documents Act (PIPEDA) provides direction on data privacy and protection. The act supports electronic communication and protection of personal information that is collected, used, or disclosed under specific circumstances. It provides comprehensive regulations on matters of privacy that pertain to breaches of security safeguards, remedies and use of electronic documents (Personal Information Protection and Electronic Documents Act). However, as of its most recent amendment of 2019, it does not cover 1) fragmented privacy and protection laws across provinces and 2) the use of algorithmic systems that hides the inner workings of the system from the user, or black-box code (Garnett 2019).

\section{Challenges and policy landscape}

The predictive accuracy of ML models is highly dependent on the diversity and complexity of the data on which it is trained (Murdoch et al 2019). Acquiring high-quality training data would require data sharing at national and international levels to provide services to patients within and across organizations (Kostkova et al 2016). We discuss examples that highlight that the power of ML models depends on the data on which it is trained. Recently, St. Michael's Hospital in Toronto used ML to predict peaks in Emergency Room (ER) admissions with 95\% accuracy. This information is used to automate and optimize ER nurse staff schedules and to reduce wait times, leading to savings of approximately $\$ 1$ million annually (Gibson 2020, Panch 2019). However, the authors in the article argue that this implementation of AI was based on a small and homogenous dataset and its effectiveness seems to be limited.

In another instance, researchers from the Vancouver General Hospital and the University of British
Columbia noted that data collected in certain departments cannot be transferred to other departments due to a lack of interoperability (To and Combes 2020).

Given the sensitivity of personal health data, its sharing requires a regulatory framework to assure its privacy. Failing to do so can jeopardize public trust (Brady 2020). Consider a recent incident on privacy invasion that led to an ongoing class-action lawsuit: patient data from the University of Chicago was shared with Google for better predictive analysis without the users' consent, and the location data from their smartphones was used to match them with their health records (Wakabayashi 2019).

Canada is the first country to adopt a national AI Strategy, the 2017 Pan-Canadian AI Strategy, and has fostered private sector innovation through the Superclusters Initiative (UNESCO 2018). Additionally, Canada released the Montreal Declaration for Responsible Development of AI, a set of ethical guidelines to initiate public dialogue and propose progressive and inclusive orientation for the future development of AI (Langlois 2019), thus playing an active role as an advocate for ethics and clear values. Furthermore, the Canadian Institute for Advanced Research aims to open a national and international forum to achieve equitable, inclusive, and ecologically sustainable AI development.

While the call for proposals is a welcome move, it remains uncertain how these recommendations will change the present state of AI in healthcare. With respect to $\mathrm{AI}$, the government of Canada promotes fairness and transparency by the way of impact assessments, public reporting, verifiability, and auditing in federal departments (Government of Canada 2019). Most recently, the Office of the Privacy Commissioner of Canada released a call for consultations to examine the state and relevancy of PIPEDA for AI and its subsequent fields (Office of the Privacy Commissioner of Canada 2020). Incorporating $\mathrm{AI}$ in healthcare is an opportunity that comes with challenges; only when AI is applied with a comprehensive policy framework will it thrive and be used ethically to protect patients. 


\section{Policy options}

i. Option 1: Maintain the status quo.

\section{Background}

In Canada, PIPEDA is central for the integration of AI and ML technologies in the private and commercial sector as it deals with disclosed personal information. It has the potential to safeguard user's privacy by defining efficient working mechanisms for the healthcare system (Office of the Privacy Commissioner of Canada 2020). Without a statutory change, in its current form, the PIPEDA will continue to be enforced at private and commercial levels, while stricter provincial laws governing health information, such as the Health Information Act in Alberta (Office of the information and Privacy Commissioner of Alberta. 2001) will take precedence.

\section{Advantages}

Standing committees can be created to review and adjust PIPEDA periodically to match the changing landscape. This intervention could help address the associated risks and concerns as and when the ML system evolves to become efficient in healthcare.

\section{Disadvantages}

In its current form, PIPEDA is primarily a commercial act; therefore, trading data is prioritized by businesses over data privacy and access to patient's data. The ambiguity of the importance of monitoring data can lead to inefficiencies with the development, implementation and sustenance of the use of AI systems between private and provincial sectors, e.g. employing a company like Google to use the ML system on patient data at a provincial hospital (Office of the Privacy Commissioner of Canada 2020). Mishandling of data can thus lead to lawsuits, incurring substantial costs to patients, hospitals, and private companies (O’Brien 2019).

ii. Option 2: Enforce the regulations laid out in Treasury Board of Canada Secretariat's Directive on Automated Decision-Making in Proposal 5 of the Office of the Privacy Commission (OPC) of Canada's consultation on AI.

\section{Background}

Bias in medicine is a recurrent concern in the healthcare community. The development of successful AI systems in tandem with a shift in the medical curriculum can help mitigate this problem. It is important that physicians understand the inherent bias that can emerge from $\mathrm{ML}$ as small inconsistencies in the data input can lead to substantial mistakes in the output (Wang et al 2020). Proposal 5 states that there should be a "Requirement for the application of Privacy by Design and Human Rights by Design in all phases of processing, including data collection" (Office of the Privacy Commissioner of Canada 2020).

Following the guidelines on $\mathrm{AI}$ and data protection (European Commission 2019), the OPC states that "before launching [AI systems] into production, [private and commercial companies should] develop processes to test for unintended data biases and other factors that may unfairly impact the outcomes. This directive (Government of Canada 2019) further requires the completion of an algorithmic impact assessment prior to the production of any automated decision system. The assessment must also be updated when system functionality, or the scope of the automated decision system changes in order to continuously monitor for and prevent such negative impacts." Using well designed empirical studies, a similar framework can be applied in the context of healthcare. These regulations can establish the sensitivity and specificity of the algorithm, thus enabling the system to move beyond the black box phenomenon (London 2019). Therefore, rendering the input, outputs, and machinery of the operation interpretable to the physicians will make the systems and their outputs easier to understand in the context of healthcare (Office of the Privacy Commissioner of Canada 2020).

\section{Advantages}

Since AI can offer objectivity in several areas of healthcare, moving beyond a black box system can help address sources of unintentional discrimination (Borocas and Selbst 2016), thus providing for greater reliability, predictability, and consistency in the diagnosis and prognosis of the patients (Institute of Medicine 2001). Objectivity and accountability can be further facilitated by using a dataset that is diversified and truly encompasses a sample of the population that represents the locality of the healthcare facilities. 


\section{Disadvantages}

The regulations in Proposal 5 of the OPC guideline do not explicitly require the AI system to be monitored (Wang et al 2020). Therefore, it will be imperative that the output remains a resource upon which the physician can reflect to determine the diagnosis and subsequent steps for treatment. As such, the physician will need to regularly review subtleties that are presently beyond the computational prowess of the algorithm and that can impact the data, such as the emotion of a given moment, the state, or the body language of the patient (Rowe 2019). As the Canadian Medical Protective Association states, it is the responsibility of the doctor to assess the usefulness of information derived from AI (Montreal Declaration for a Responsible Development of Artificial Intelligence 2018).

\section{iii. Option 3: Amend and stringently implement PIPEDA} at the federal level

\section{Background}

In Canada, national regulation is not always uniformly implemented across subnational governance systems. The provinces of Quebec, Alberta, Ontario, and British Columbia are not under the jurisdiction of the PIPEDA but instead have provincial laws that are like or stricter than PIPEDA (Office of the Privacy Commissioner of Canada 2018). Presently in Canada, laws governing hospitals are left to the jurisdiction of the province. PIPEDA can only be implemented on hospital information when or if it is transferred, after which provincial laws take precedence (Office of the Privacy Commissioner of Canada 2015). This multi-step process has the potential to create inefficiency and loopholes for data privacy. Concurrently, the present regulations state that Electronic Health Records (EHR) cannot be transferred across provinces (Goldman 2019).

To tackle the issue of data privacy and consent, we propose the following amendments to PIPEDA with respect to healthcare and AI systems:

- Allow for digital interoperability and access to electronic health records across provinces. This approach would be similar to the EU's recent recommendation for an electronic health record exchange format across EU member states (European Commission 2019) and the USA's Fast Healthcare Interoperability Resources (FHIR) standards for EHR (Bresnick 2016).

- Companies that have access to Canadian health information (raw or processed data) for manufacturing and training AI systems for healthcare should follow PIPEDA. The transmission of such information should take place through a Canadian server to stay under Canadian jurisdictions (Callaghan 2018, Coos 2019).

- Create a national-level task force that will foresee the development of these guidelines and their application. This task force can work in liaison with provincial leaders to acquire reports on the execution and performance of these laws.

\section{Advantages}

With consistent policies across the country on data privacy and sharing, digital interoperability can facilitate the exchange of healthcare data across different delivery points within the country. For example, patients can access their own diagnostic and treatment plans, empowering them to ask for second opinions and, thus, making them active managers of their health. Accessing one's personal health data creates trust in digital technologies and better patient-doctor relations (Lehne et al 2019).

\section{Disadvantages}

Interoperability and the ability to access and share data with different sectors come with concerns about patient data privacy and misdiagnosis. The concerns about data security and privacy discourage physicians from using ML when providing care for patients (Vogel 2019). These concerns need to be addressed by establishing a rigorous structure that disseminates the ideologies implemented by $\mathrm{AI}$ in healthcare and interoperability. ${ }^{1}$ Knowledge dissemination can be facilitated through science communication (e.g. public consultations) and outreach programs. Another main concern is that this

\footnotetext{
1The training and implementation of algorithms in the healthcare system will be standardized across the nation. The outcomes of the EU's EHR policy could be monitored to shed light on caveats and strengths of this system as it is adopted for Canada.
} 
option could lead to loss of privacy for patient data if PIPEDA overrules stricter provincial laws.

iv. Option 4: Establish a mandatory amendment for provincial laws with respect to healthcare to minimally uphold the data privacy standards of the General Data Protection Regulation (GDPR) framework

\section{Background}

As stated earlier, the provinces of Quebec, Alberta, Ontario, and British Columbia are not under the jurisdiction of the PIPEDA but instead have provincial laws that are similar to or stricter than PIPEDA (Office of the Privacy Commissioner of Canada 2018). Currently, Quebec is in the process of modifying its act on the protection of personal information in the private sector to encompass the rules laid out in the GDPR, which is a framework followed in the European Union (EU) (General Data Protection Regulation 2016). However, it remains uncertain if other provinces and territories will uphold the GDPR or stricter frameworks in their provincial regulations (Modernizing Canada's Privacy Act 2020).

\section{Advantages}

As federal laws cannot take precedence over provincial jurisdictions in healthcare in Canada (Government of Canada 2020) the uniformity of norms at the provincial level will enable crossprovincial accountability when data is shared. This option will be cost-effective and easiest to implement as provincial norms will be similar.

\section{Disadvantages}

The disparities in the economic status of a province or territory, available healthcare facilities, and infrastructure could act as barriers for provinces to uphold GDPR regulations (Walberg 2008, Allen 2008).

\section{Policy recommendations}

AI and ML technologies can offer physicians important insight concerning a patient's medical needs. However, with respect to Proposal 5 of the OPC's consultation on AI, input for ML systems in healthcare will require a significant quantity and quality of datasets to mitigate bias in the algorithmic systems. In addition, reliable analysis to provide proper treatments to patients is highly dependent on data sharing amongst provinces and/or territories in Canada.

A main concern of the implementation of the amendments to PIPEDA as brought up in Option 3 is the possibility of privacy breaches if PIPEDA were to overrule stricter provincial laws that are currently preceding. For AI and ML to thrive in healthcare in Canada, privacy policies need to uphold the different challenges that come with such technologies. It is necessary that PIPEDA includes specific provisions regarding the protection of patient medical records or private information. As such, data inputs and access to patients' confidential data should be regulated to assure that patients give their full consent to physicians to disclose their personal information.

We therefore recommend that there be an open discussion between federal and provincial legislators to initiate cooperation between jurisdictions to protect personal medical information no matter where or to whom the data is shared. Given that different jurisdictions apply in Canada for the healthcare system, this common ground at the provincial and federal level will allow a minimum standard nationwide for data privacy. As such, we believe that the combined execution of Option 2 and Option 4 will enable unprecedented growth of $\mathrm{AI}$ and ML in healthcare by placing uniform privacy regulations among different sectors of healthcare. This enhanced collaboration between companies, physicians and governments at the provincial level can then make way for a federal level implementation of privacy regulations regarding patients' confidential data, a point that must be addressed if Option 3 were to be implemented.

Considering the growing need for harmonized health data collection and privacy regulations for patient records, the combined implementation of Option 2 and Option 4 can increase digital interoperability as well as accountability amongst different stakeholders. 


\section{References}

Allen, S. Does Equity in Healthcare Use Vary across Canadian Provinces? Healthcare policy. 2008. https://www.ncbi.nlm.nih.gov/pmc/articles/PM C2645154/

Automatic Processing of Personal Data. Directorate General of Human Rights and Rule of Law. 2019. https://rm.coe.int/168063e391

Barocas, S., Selbst AD., Big Data's Disparate Impact. SSRN. 2016.

https://papers.ssrn.com/sol3/papers.cfm?abstra ct id=2477899

Brady M., Interoperability rule exposes gaps in protecting privacy. in Modern Healthcare 2020. https://www.modernhealthcare.com/informatio $\underline{\text { n-technology/interoperability-rule-exposes- }}$ gaps-protecting-privacy

Bresnick J., 4 Basics to Know about the Role of FHIR in Interoperability. in Health IT Analytics. 2016. https://healthitanalytics.com/news/4-basics-toknow-about-the-role-of-fhir-in-interoperability

Callaghan, S., PIPEDA: What Canadian businesses need to know,

2018.https://www.cira.ca/blog/cybersecurity/pi peda-what-canadian-businesses-need-know

Cattell, J., Chlukuri. S., Levy, M., How big data can revolutionize pharmaceutical R\&D. Mckinsey \& Company. 2013. https://www.mckinsey.com/industries/pharmac euticals-and-medical-products/ourinsights/how-big-data-can-revolutionizepharmaceutical-r-and-d

Consultation on the OPC's Proposals for ensuring appropriate regulation of Artificial intelligence, Office of the privacy commissioner of Canada, 2020 https://www.priv.gc.ca/en/about-theopc/what-we-do/consultations/consultationai/pos ai 202001/

Coos, A., PIPEDA vs GDPR: The Key Differences. 2019, Endprint protector. https://www.endpointprotector.com/blog/piped a-vs-gdpr-the-key-differences/

European Commission, European Electronic Health Record exchange format. 2019. https://ec.europa.eu/digital-singlemarket/en/news/recommendation-europeanelectronic-health-record-exchange-format

Future AI Opportunities for Improving Care Delivery, Cost, and Efficacy, in Health IT Analytics 2019. https://healthitanalytics.com/news/future-aiopportunities-for-improving-care-delivery-costand-efficacy
Garnett, D. What Happened to the Distinction Between Primary and Secondary Research? 2019. https://douggarnett.com/uncategorized/ai-bigdata-question-what-happened-to-the-distinctionbetween-primary-and-secondary-research/.

General Data Protection Regulation. 2016. https://gdprinfo.eu

Gibson, G., Innovation in healthcare depends on responsible, expanded data access for AI researchers, in The Globe and Mail 2020. https://www.theglobeandmail.com/opinion/arti cle-innovation-in-health-care-depends-onresponsible-expanded-data-access/

Goldman, B., A National Electronic Health Record For All Canadians, in 2019. https://www.cbc.ca/radio/whitecoat/a-nationalelectronic-health-record-for-all-canadians$\underline{1.4976932}$

Government of Canada. Directive on Automated DecisionMaking. 2019. https://www.tbs-sct.gc.ca/pol/doceng.aspx?id=32592

Health Information Act. Office of the information and Privacy Commissioner of Alberta. 2001 https://www.alberta.ca/health-informationact.aspx

Institute of Medicine, Committee on Quality of Healthcare, Crossing the Quality Chasm: A New Health System for the 21st Century, 2001. https://pubmed.ncbi.nlm.nih.gov/25057539/

Kostkova, P., H. Brewer, S. de Lusignan, E. Fottrell, B. Goldacre, G. Hart, P. Koczan P. Knight, C. Marsolier, R. A. McKendry, E. Ross, A. Sasse, R. Sullivan, S. Chaytor, O. Stevenson, R. Velho, and J. Tooke. "Who Owns the Data? Open Data for Healthcare." Front Public Health 4: 7.2016. https://www.ncbi.nlm.nih.gov/pmc/articles/PM C4756607/

Langlois, S., Developing AI in a responsible way, in UDEM NOUVELLES 2018, https://phys.org/news/201812-ai-responsible.html

Lehne, M., et al, Why digital medicine depends on interoperability. NPJ Digit Med, 2019. 2: p. 79. https://www.nature.com/articles/s41746-0190158-1

London, AJ., Artificial Intelligence and Black-Box Medical Decisions: Accuracy versus Explainability. The Hastings Centre Report. 2019. https://onlinelibrary.wiley.com/doi/10.1002/ha st. 973

Luxton, D.D., Artificial Intelligence in Behavioral and Mental Healthcare. 1 ed. 2016: Academic Press. https://www.sciencedirect.com/book/97801242 $\underline{02481 / a r t i f i c i a l-i n t e l l i g e n c e-i n-b e h a v i o r a l-a n d-~}$ $\underline{\text { mental-health-care }}$ 
Martin, N., Artificial Intelligence Is Being Used To Diagnose Disease And Design New Drugs, in Forbes. 2019. https://www.forbes.com/sites/nicolemartin1/2 019/09/30/artificial-intelligence-is-being-usedto-diagnose-disease-and-design-new-drugs/

Modernizing Canada's Privacy Act. 2020. https://www.justice.gc.ca/eng/csj-sjc/palprp/modern.html

Montréal Declaration for a Responsible Development of Artificial Intelligence 2018. https://recherche.umontreal.ca/english/strategi c-initiatives/montreal-declaration-for-aresponsible-ai/

Murdoch, W. J., C. Singh, K. Kumbier, R. Abbasi-Asl, and B. Yu. Definitions, methods, and applications in interpretable machine learning. Proc Natl Acad Sci USA116(44):22071-22080. 2019. https://www.pnas.org/content/116/44/22071

News, S., SFU alumni company MetaOptima taps into Australian dermatology market, in SFU news 2018, SFU.4.

https://www.sfu.ca/sfunews/stories/2018/07/s fu-alumni-company-metaoptima-taps-intoaustralian-dermatology.html

O’Brien C., LifeLabs hack: Data protection 'a big problem for a lot of companies', in CTV News. 2019. https://www.ctvnews.ca/canada/lifelabs-hackdata-protection-a-big-problem-for-a-lot-ofcompanies-1.4748607

O'Kane, J., AI startup enlisted for project to predict suicide risk in Canada, in The Globe and mail 2018. https://www.theglobeandmail.com/technology/ government-enlists-ai-startup-for-project-topredict-suicide-risk-in-canada/article37475839/

Office of the Privacy Commissioner of Canada. Summary of privacy laws in Canada, Canada, Editor. 2018.https://www.priv.gc.ca/en/privacytopics/privacy-laws-in-canada/02 05 d 15/

Panch, T., Mattie, H., Celi, LA., The "inconvenient truth" about AI in healthcare. Npj Digital Medicine. 2019. https://www.nature.com/articles/s41746-019$\underline{0155-4}$

Rowe, J., How AI could help the doctor-patient relationship. AI Powered Healthcare, 2019. Modernizing Canada's

PrivacyAct.https://www.justice.gc.ca/eng/csjsjc/pa-lprp/modern.html

Siwicki, B., Northwell, UCSF, UNC using chatbot and related tech to manage COVID-19 patients, in Healthcare IT News. 2020. https://www.healthcareitnews.com/news/north well-ucsf-unc-using-chatbot-and-related-techmanage-covid-19-patients
Steig, C., How this Canadian start-up spotted coronavirus before everyone else knew about it, in make it. 2020.

https://www.cnbc.com/2020/03/03/bluedotused-artificial-intelligence-to-predictcoronavirus-spread.html

The Application of PIPEDA to Municipalities, Universities, Schools, and Hospitals, Office of the privacy commissioner of Canada, Canada, Editor. 2015. https://www.priv.gc.ca/en/privacytopics/privacy-laws-in-canada/the-personalinformation-protection-and-electronicdocuments-act-pipeda/r_o_p/02 05 d 25/To W., Combes P., Leveraging AI and Machine Learning to Advance Interoperability in Healthcare. in Hit Consultant. 2020. https://hitconsultant.net/2020/01/15/leveragin g-ai-and-machine-learning-to-advanceinteroperability-in-healthcare/

UNESCO. Canada first to adopt a strategy for artificial intelligence.

2018.

http://www.unesco.org/new/en/mediaservices/single-

view/news/canada first to adopt strategy for a rtificial intelligence/Vogel, L. Rise of medical AI poses new legal risks for physicians. CMAJ. 2019. https://www.cmaj.ca/content/191/42/E1173

Wakabayashi, D., Google and the University of Chicago Are Sued Over Data Sharing, in New York Times 2019. https://www.nytimes.com/2019/06/26/technol ogy/google-university-chicago-data-sharinglawsuit.html

Walberg, R. Which provinces deliver the best health care. Frontier Center for Public library. 2008. https://fcpp.org/2008/09/17/which-provincesdeliver-the-best-healthcare/

Wang F., Kaushal R., Khullar D., Should Health Care Demand Interpretable Artificial Intelligence or Accept "Black Box" Medicine?, Annals of Internal Medicine. 2020.https://www.acpjournals.org/doi/10.7326 /M19-2548

Wolff, J,. Pauling, J., Keck, A., Baumbach, J., The Economic Impact of Artificial Intelligence in Health Care: Systematic Review. Journal of Medical Internet Research. 2020 . https://www.jmir.org/2020/2/e16866/ 
Sumedha Sachar is a Postdoctoral fellow at University of Montreal, Department of Pharmacology. Her research focuses on liposome mediated miRNA delivery to Glioblastoma in mouse models. She is also a volunteer with non-profit organization Science and Policy Exchange.

Contact: sumedhasachar@gmail.com

Maïa Dakessian is a Master's student in Bioethics, at the Université de Montréal, Département de médecine sociale et préventive. She is interested in neuroethics and how language is represented in the brain. She holds an undergraduate degree in Cognitive Neurosciences as well as one in Linguistics and Psychology, both from the Université de Montréal. For the past year, Maïa has been a volunteer with the non-profit organization Science \& Policy Exchange. As the editor for the SPE Medium since December 2019, she coordinates writing ideas and projects to promote the importance of science in policy-making. Contact: maia.dakessian@umontreal.ca

Saina Beitari is a graduate student at McGill University, department of Microbiology and Immunology. As a PhD student her research focused on studying the interaction between innate immunity and various viruses including HIV and SARS-COV-2. Saina is a volunteer with the non-profit organization Science \& Policy Exchange. She is also a board member of AIDS Community Care Montreal.

Contact: saina.beitari@mail.mcgill.ca

Saishree Badrinarayanan is a PhD candidate in the Integrated Program in Neuroscience at McGill University. She holds a Master of Science from the University of Edinburgh in Integrative Neuroscience. Her research work focuses on the circuits responsible for spatial navigation and memory in mice. Saishree currently serves as treasurer for the student-led non-profit organization Science \& Policy Exchange.

Contact: saishree.badrinarayanan@mail.mcgill.ca

\section{Acknowledgements}

The authors would like to thank Dr. Sam Garnett, Anh-Khoi Trinh and Shawn McGuirk from Science \& Policy Exchange for comments and suggestions during the development of this policy memo. They would also like to thank their editors Linda D. Ho and Derek Wang for feedback during the revisions.

\section{Disclaimer}

The authors declare no conflict of interest. 